\title{
A Method for Estimating the Storage Life of Detonation Bomb Based on Weibull Distribution.
}

\author{
Yifan Song ${ }^{a}$, Minghua Chen, Yunxian Jia and Weijia Huang \\ Army Engineering University of PLA, Shijiazhuang 050003, China. \\ acelticsong@126.com
}

Keywords: detonation bomb, storage life, success-or-failure test, parameter estimation.

\begin{abstract}
This paper explains and analyzes the failure characteristics of detonation projectile in storage condition, and demonstrates a mathematical model for the life assessment of detonation projectiles based on the test data which is established. Based on the test data of success-or-failure, the storage reliability life model of the DFB91-35 detonation bomb was evaluated. When the confidence level is $90 \%$ and the lower limit of reliability is 0.90 , the storage life of the detonation bomb based on the model is 8.25 , which is consistent with the actual situation.
\end{abstract}

\section{Introduction}

Detonation is usually equipped with the public security and armed police forces, can use its huge deterrent effect to disperse the crowd, control the situation and quell riots.It can minimize the casualties and protect state property from huge losses. It is also an application in armed forces on duty and disposal emergencies extensive non-lethal weapons. The grass-roots units must have a certain amount of reserves at ordinary times.

In the storage state of detonation bombs ammunition is not working, but subject to the role of environmental stress, which makes the various metamorphic phenomenon happen on components of ammunition such as corrosion, aging, decomposition,etc., the ammunition performance degradation, lower reliability. Moreover, as the storage time goes on, the metamorphic phenomenon will become more and more serious, so that the ammunition can not complete its prescribed function and become invalid. This kind of environmental stress and time double factor induced failure, because the storage time of ammunition is very long and the working time is relatively short, so the ammunition storage failure is particularly remarkable.

So after a long-term storage, detonation can be effectively used in the line of duty, the reliability assessment and life prediction is a concern, for improving the ammunition storage reliability design, improve the production quality, and improve ammunition storage of ammunition quality monitoring is of great significance.

\section{The Concept of Detonation Bombs}

The DFB91-35 detonation projectile studied in this paper can be launched by two kinds of 35 millimeter riot grenade launchers with shoulder shots and guns. It is mainly used to dispel, prevent unlawful crowds and riots within the range of 75-300 meters, and subdued criminals hidden in the building.

The whole body of detonation bomb is assembled by the warhead and cartridge components, and is mainly made up of 4 parts, namely, the firing mechanism, the arming device, the projectile and the main charge.

The failure modes mainly include dismembered, projectile, early-explosion, half-explosion, misfire, disintegration of warhead, the unqualified shooting accuracy, the poor matching and the storage difference.

The main factors that affect the storage reliability and life of detonation bomb are: failure of firing mechanism, corrosion and deformation of projectile, deformation of delay tube and failure of propellant. 


\section{Mathematical Model}

\subsection{Reliability Function}

We generally analyse the physical properties of the machinery or electronics, as combustion mechanism, insurance device and projectile, then conclude that the characteristics of their life distributions. Besides, the main loading part can be based on the characteristics of its chemical properties analysis of life.[1] Therefore, it can be determined that the life of detonation is obeying the two-parameter weibull distribution.[2]

Its distribution function is:

$$
F(t)=1-\exp \left(-\frac{t}{\beta}\right)^{m} t \geq 0
$$

The distribution density function is:

$$
f(t)=\frac{m}{\beta} t^{m-1} \exp \left(-\frac{t}{\beta}\right)^{m}
$$

The reliability function is:

$$
R(t)=1-F(t)=\exp \left(-\frac{\mathrm{t}}{\beta}\right)^{m}
$$

In the above equation, $t$ is the time variable; $m>0$, for shape parameter; $\beta>0$, for scale parameter.

\subsection{Data Structure of Success or Failure Test}

Because the ammunition reliability test is a success or failure test, the failure of ammunition is not accurately observed. When the ammunition is stored for $t$ years, only the ammunition is known to have failed or still available. And the former only indicates that the failure occurred before $t$, but we can not know the exact time, and the latter can not predict the remaining life either.[3]

Therefore, when $\mathrm{X}$ represents the storage life of ammunition, $\mathrm{X}$ cannot be directly observed, only Y can be observed.

$$
Y= \begin{cases}1 & \text { if } X \leq t \\ 0 & \text { if } X>t\end{cases}
$$

' 0 ' represents the use of ammunition; ' 1 ' represents the failure of ammunition.

Therefore, the success or failure data structure can be expressed as:

$$
\left(t_{i}, n, f_{i}\right)(i=1,2, \ldots, k)
$$

In the formula (5): $t_{i}$ is the ammunition storage time, $\mathrm{n}$ is the number of samples per test, $f_{i}$ is the number of failures in $\mathrm{n}$ rounds, and $\mathrm{k}$ is the number of detection time points.

In this experiment, the storage life of the jth sample in group I was $T_{i j}(\mathrm{i}=1,2 \ldots, \mathrm{k} ; \mathrm{j}=1,2, \ldots \mathrm{n})$, $T_{i j}$ independently identically distributed in two-parameter weibull distribution $F\left(t_{i} ; m, \beta\right)$. We can make:

$$
Y_{i j}= \begin{cases}1 & \text { if } T_{i j} \leq t_{i} \\ 0 & \text { if } T_{i j}>t_{i}\end{cases}
$$

Then, $Y_{i j}$ is distributed separately from (0-1) distribution, so:

$$
f_{i}=\sum Y_{i j} \sim B\left(n, p_{i}\right)
$$


In the last-written denominator, $p_{i}=\left(t_{i} ; m, \beta\right)$.That is, the number of failure $f_{i}$ in each experimental sample is subordinate to the binomial distribution of $n$ and $p_{i}$.

\section{Parameter Estimation}

In order to determine the reliable storage life distribution of detonation projectile, we need to estimate the parameter value of the distribution.A large number of examples show that the least square method and the maximum likelihood method can be used to estimate the parameter values of the distribution, but the least square method is less effective. Therefore, in this paper, the maximum likelihood estimation method is adopted to estimate the parameters. [4] According to the experimental $\operatorname{data}\left(t_{i}, n, f_{i}\right)$, the corresponding likelihood function is obtained:

$$
L(m, \beta)=\prod_{i=1}^{c}\left(\begin{array}{c}
n_{i} \\
f_{i}
\end{array}\right) p_{i}^{f_{i}}\left(1-p_{i}\right)^{n_{i}-f_{i}}
$$

Because $\left(\begin{array}{c}n_{i} \\ f_{i}\end{array}\right)$ is the constant, does not affect the distribution of extreme value point, can be omitted. We substituted $p_{i}=\left(t_{i}, m, \beta\right)$ into the formula $(8)$, and the logarithm of the logarithm can be obtained as follows:

$$
\ln L(m, \beta)=\sum_{i=1}^{k}\left\{f_{i} \ln F\left(t_{i} ; m, \beta\right)+\left(n_{i}-f_{i}\right) \ln \left(1-F\left(t_{i} ; m, \beta\right)\right)\right\}
$$

Substitute double parameters weibull distribution function formula on the formula (9), its maximum points, can get the unknown parameter $\mathrm{m}, \beta$ maximum likelihood estimation of $\widehat{m}, \hat{\beta}$.

\section{Reliable Life Prediction.}

At the $(1-\alpha)$ confidence level, the confidence lower limit is determined by the following formula:

$$
\sum_{i=1}^{k} C_{n_{i}}^{f_{i}}\left(1-R_{L}\right)^{f_{i}} R_{L}^{n_{i}-f_{i}}=\alpha
$$

$R_{L}$ Represents the lower limit of ammunition reliability. The formula (10) indicates that $R_{L}$ is sufficient to ensure that the event 'fails to reach $f_{i}$ ' will not be a small probability event, and the probability of occurrence will not be lower than the small probability. In $n$ tests, if there are no failed detonation bombs, there are:

$$
R_{L}=\alpha^{1 / \mathrm{n}}
$$

When $R_{L}$ is given to timing, the storage of reliable life $T_{R}$ meets:

$$
\mathrm{P}\left[R\left(T_{R}\right) \geq R_{L}\right]=1-\alpha
$$

According to reliability function, weibull distribution can be obtained under the flash bang estimates of the storage life of the $\widehat{T}_{R}$ :

$$
\widehat{\mathrm{T}}_{R}=\hat{\beta} \cdot\left[\ln \left(\frac{1}{R_{L}}\right)\right]^{\frac{1}{m}}
$$




\section{Case Analysis}

In the first half of 2018, the test of quality identification of anti-riot ammunition was carried out. In the experiment, the performance tests of 120 samples of $15 \mathrm{a}, 14 \mathrm{a}, 13 \mathrm{a}, 11 \mathrm{a}$ and $9 \mathrm{a}$ were extracted relatively.

Test items include 8 projects. Inspection of the product packaging, check product appearance and size, decomposition inspection, the delay time test, reliability test, maximum range test, precision test, power test test, etc. The first three are static and the last five are field tests.

The problems found in the static test mainly include the leakage of the aluminum plastic bags, the oversized size and the wrinkling of the warhead surface, which may affect the product performance. In the field test, the problem of upper limit of shooting accuracy and early explosion is presented.

The results of test failures are shown in table 1.

Table 1. Test Results of Detonation Bomb Storage Reliability.

\begin{tabular}{cccccc}
\hline Items & Scheme 1 & Scheme 2 & Scheme 3 & Scheme 4 & Scheme 5 \\
\hline Storage life /a & 15 & 14 & 13 & 11 & 9 \\
Failure numbers & 11 & 9 & 8 & 6 & 2 \\
\hline
\end{tabular}

In the above data, the iterative calculation can be weibull distribution function of the shape parameter $\widehat{m}$ and scale parameters $\hat{\beta}$.

\section{Conclusion}

In this paper, the storage life of detonation bomb is evaluated based on the test data of the success or failure of the storage life test of detonation bomb. The evaluation result is $8.25 \mathrm{a}$, indicates that when the lower limit of reliability is $90 \%$, the storage time of detonation bomb is $8.25 \mathrm{a}$, and the detonation bomb with a reliability of $90 \%$ is still qualified.

The paper also reflects in the normal environment under stress and time stress levels, and ensures the armed police forces at the grass-roots level can be used by the detonation of ammunition storage life reached the standard of factory ( $8 \mathrm{a}$ ). So there is no need for completely destroyed. By improving inventory conditions, all units can extend the storage life of the detonation bomb as long as possible, thereby reducing unnecessary waste.

According to the mathematical model established in this paper, the storage life of some batch detonation bombs is verified, which proves that the evaluation results are in accordance with the actual conditions.

\section{References}

[1]. Zhu Feng-chun, Wang Xiao-ming, Cui Da-wei, et al. An Evaluation Method of Reliable Stockpile Lifetime of Air Ammunition. Journal of Ballistics. Vol.19 (2007) No.1, p, 68-71.

[2]. Li Ming-lun, Li Dong-yang, ZHENG Bo, Ammunition Storage Reliability, National Defence Industry Press, 1997.

[3]. Zheng Bo, Li Gan, Li Ming. Evaluation of Ammunition Storage Life Based on Test Data of Success-failure Type. Journal of Ordnance Engineering College. Vol.17 (2005) No. 5, p.29-30.

[4]. Gao Meng, Wang Jin-zhu, He Xue-guang. Evaluation of Guided Ammunition Storage Life Based on Reliability Statistics. Equipment Environmental Engineering. Vol.10 (2013) No.4, p.110-113. 